OPEN ACCESS

Edited by:

Catherine Sautes-Fridman, INSERM U1138 Centre de Recherche des Cordeliers, France

Reviewed by:

Daniel E. Speiser,

Université de Lausanne, Switzerland

Cecile Gouttefangeas,

University of Tübingen, Germany

*Correspondence:

Rouzheng Wang

wrz8526@vip.163.com

Ning Li

liningbjyah@vip.sina.com

Tao Dong

tao.dong@imm.ox.ac.uk

tThese authors have contributed equally to this work

Specialty section

This article was submitted to Cancer Immunity and Immunotherapy,

a section of the journal Frontiers in Oncology

Received: 14 May 2019 Accepted: 30 September 2019 Published: 25 October 2019

Citation:

Li X, Wang R, Fan P, Yao X, Qin L, Peng Y, Ma M, Asley N, Chang X,

Feng $Y$, Hu Y, Zhang Y, Li C,

Fanning $G$, Jones $S$, Verrill $C$,

Maldonado-Perez D, Sopp P,

Waugh C, Taylor S, Mcgowan S, Cerundolo V, Conlon C, McMichael A, Lu S, Wang X, Li N and Dong T (2019)

A Comprehensive Analysis of Key

Immune Checkpoint Receptors on

Tumor-Infiltrating T Cells From Multiple

Types of Cancer.

Front. Oncol. 9:1066.

doi: 10.3389/fonc.2019.01066

\title{
A Comprehensive Analysis of Key Immune Checkpoint Receptors on Tumor-Infiltrating T Cells From Multiple Types of Cancer
}

\begin{abstract}
Xi Li ${ }^{1,2,3+}$, Rouzheng Wang ${ }^{1,2,4 * t}$, Peiwen Fan ${ }^{1,4}$, Xuan Yao ${ }^{2,3}$, Ling Qin ${ }^{5}$, Yanchun Peng ${ }^{2,3}$, Miaomiao Ma ${ }^{1,4}$, Neil Asley ${ }^{6}$, Xuimei Chang ${ }^{1,4}$, Yaning Feng ${ }^{1,4}$, Yunhui Hu ${ }^{1,4}$, Yonghong Zhang ${ }^{5}$, Chris $\mathrm{Li}^{7}$, Gregory Fanning ${ }^{7}$, Stephanie Jones ${ }^{8}$, Clare Verrill ${ }^{9}$, David Maldonado-Perez ${ }^{9}$, Paul Sopp ${ }^{10}$, Craig Waugh ${ }^{10}$, Stephen Taylor ${ }^{11}$, Simon Mcgowan ${ }^{11}$, Vincenzo Cerundolo ${ }^{2,3}$, Christopher Conlon ${ }^{2}$, Andrew McMichael ${ }^{2}$, Shichun Lu ${ }^{12}$, Xiyan Wang ${ }^{1,4}$, Ning $\mathrm{Li}^{5 * t}$ and Tao Dong ${ }^{2,3 * t}$
\end{abstract}

${ }^{1}$ Key Laboratory of Tumor Immunology and Radiation Therapy, Third Affiliated Hospital, Xinjiang Tumor Hospital, Chinese Academy of Medical Sciences (CAMS), Xinjiang Medical University, Ürümqi, China, ${ }^{2}$ Nuffield Department of Medicine (NDM), Chinese Academy of Medical Sciences Oxford Institute (CAMS Oxford Institute), University of Oxford, Oxford, United Kingdom, ${ }^{3}$ MRC Human Immunology Unit, Radcliffe Department of Medicine, Weatherall Institute of Molecular Medicine, University of Oxford, Oxford, United Kingdom, ${ }^{4}$ Third Affiliated Hospital, Xinjiang Tumor Hospital, Xinjiang Medical University, Ürümqi, China, ${ }^{5}$ Beijing You'an Hospital, Capital Medical University, Beijing, China, ${ }^{6}$ Single Cell Genomics Facility, Weatherall Institute of Molecular Medicine, University of Oxford, Oxford, United Kingdom, ${ }^{7}$ China R\&D, Janssen

Pharmaceuticals, Shanghai, China, ${ }^{8}$ Oxford Radcliffe Biobank, Department of Cellular Pathology, Oxford University Hospitals NHS Trust, Oxford, United Kingdom, ${ }^{9}$ Nuffield Department of Surgical Sciences, NIHR Oxford Biomedical Research Centre, University of Oxford, Oxford, United Kingdom, ${ }^{10}$ Flow Cytometry Facility, Weatherall Institute of Molecular Medicine, University of Oxford, Oxford, United Kingdom, ${ }^{11}$ Bioinformatics Team, Weatherall Institute of Molecular Medicine, University of Oxford, Oxford, United Kingdom, ${ }^{12}$ China Military General Hospital, Beijing, China

Background: Cancer patients often display dysfunctional antitumor T-cell responses. Because noteworthy benefits of immune checkpoint pathway blockade, such as programmed cell death protein 1 (PD-1) inhibitors, have been achieved in multiple advanced cancers, the next critical question is which mono-blockade or combinatorial blockade regimens may reinvigorate antitumor T-cell immunity in those cancer patients while limiting immune-related adverse effects.

Method: This study recruited, in total, 172 primary cancer patients (131 were blood-tumor-matched patients) who were treatment-naïve prior to the surgeries or biopsies covering the eight most prevalent types of cancer. With access to fresh surgical samples, this study simultaneously investigated the ex vivo expression level of eight known immune checkpoint receptors [PD-1, cytotoxic T-lymphocyte antigen-4 [CTLA-4], T-cell immunoglobulin and mucin-domain containing-3 [Tim-3], 2B4, killer cell lectin like receptor G1 [KLRG-1], TIGIT, B- and T-lymphocyte attenuator [BTLA], and CD160] on tumor-infiltrating T cells (TILS) and paired circulating T cells in blood from a 131-patient cohort.

Results: We found increased an expression of PD-1 and Tim-3 but a decreased expression of BTLA on TILS when compared with peripheral blood from multiple types of cancer. Moreover, our co-expression analysis of key immune checkpoint receptors delineates "shared" subsets as PD-1+Tim-3+TIGIT+2B4+KLRG-1-CTLA-4- and 


\begin{abstract}
PD-1+TIGIT+2B4+Tim-3-KLRG-1-CTLA-4- from bulk CD8 TILs. Furthermore, we found that a higher frequency of advanced differentiation stage $T$ cells (CD27-CCR7-CD45RA-) among the "shared" subset (PD-1+Tim-3+TIGIT+2B4+KLRG-1-CTLA-4-) in bulk CD8 TILs was associated with poorly differentiated cancer type in cervical cancer patients.
\end{abstract}

Conclusions: To our knowledge, our study is the first comprehensive analysis of key immune checkpoint receptors on T cells in treatment-naïve, primary cancer patients from the eight most prevalent types of cancer. These findings might provide useful information for future design of mono-blockade/combinatorial blockades and/or genetically modified T-cell immunotherapy.

Keywords: T cells, inhibitory receptor, tumor-infiltrating lymphocytes, tumor microenvironment, combinatorial checkpoint blockade

\section{INTRODUCTION}

The combinations of co-stimulatory and co-inhibitory receptors on $\mathrm{T}$ cells enable effective T-cell responses to viruses and tumors while also limiting immunopathology and autoimmunity $(1,2)$. However, this homeostasis can be disrupted by persistent antigen stimulation and immunosuppressive mediators released in the tumor microenvironment, leading $\mathrm{T}$ cells to a dysfunctional state, known as T-cell exhaustion, which fails to control viruses, or cancer (3-5). Intensive studies of dysfunctional $\mathrm{T}$ cells in cancers and in the context of chronic virus infection-for instance, with hepatitis B virus (HBV), hepatitis C virus (HCV), and human immunodeficiency virus (HIV)-suggested that exhausted $\mathrm{T}$ cells are characterized by an elevated expression of single or multiple inhibitory receptors (IRs) along with dysfunctional production of cytokines and activation of apoptotic signaling (6-10). However, the fundamental mechanism by which $\mathrm{T}$ cells become dysfunctional in the tumor microenvironment remains unclear.

In 2011, ipilimumab became the first approved blockade agent targeting the T-cell inhibitory receptor "cytotoxic T-lymphocyte antigen-4" (CTLA-4); advanced melanoma patients treated with ipilimumab demonstrated improved tumor control and survival (11). More recently, checkpoint blockade immunotherapy

\footnotetext{
Abbreviations: 2B4, CD244; ALK, Anaplastic lymphoma kinase; BRC, Biomedical Research Center; BSA, Bovine serum albumin; BTLA, B- and T-lymphocyte attenuator; CAMS, Chinese Academy of Medical Sciences; CCR7, C-C chemokine receptor type 7; CTLA-4, Cytotoxic T-lymphocyte antigen-4; EDTA, Ethylenediaminetetraacetic acid; EGFR, Epithelial growth factor receptor; FACS, Fluorescence-activated cell sorting; FDA, Federal Food and Drug Administration; FMO, Fluorescence minus one; HBV, Hepatitis B virus; HCC, Hepatocellular carcinoma; HCV, Hepatitis C virus; HIV, Human immunodeficiency virus; IR, Inhibitory receptor; KLRG-1, Killer cell lectin like receptor G1; NDM, Nuffield Department of Medicine; NIAID, National Institute of Allergy and Infectious Diseases; NIHR, National Institute for Health Research; NSCLC, Nonsmall-cell lung cancer; ORB, Oxford Radcliffe Biobank; ORR, Overall response rate; OxTREC, Oxford Tropical Research Ethics Committee; PBMC, Peripheral blood mononuclear cell; PBS, Phosphate-buffered saline; PD-1, Programmed cell death protein 1; SPICE, Simplified Presentation of Incredibly Complex Evaluations; TAA, Tumor-associated antigen; TCR, T-cell receptor; TIGIT, T-cell immunoglobulin and ITIM; TILs, Tumor-infiltrating lymphocytes; Tim-3, T-cell immunoglobulin and mucin-domain containing-3.
}

targeting "programmed cell death protein 1" (PD-1) has shown promise in treating multiple solid tumors, including metastatic melanoma, non-small-cell lung cancer (NSCLC), kidney cancer, urothelial cancer, head and neck cancer, and liver cancer (1216). Consequently, the Federal Food and Drug Administration (FDA) in the USA accelerated the approval of clinical trials of PD-1 blockade in multiple cancers to benefit different types of advanced-cancer patients $(15,17,18)$. Furthermore, PD-L1, as one of the ligands of PD-1, is capable of binding CD80 on activated $\mathrm{T}$ cells mediating inhibitory signaling $(19,20)$. Therefore, blocking PD-L1 with or without the co-blockade of $\mathrm{PD}-1$ is a promising approach to restore the dysfunctional $\mathrm{T}$ cells in cancers (2). In fact, PD-L1 blockade has shown durable responses to induce tumor regression in patients with multiple cancers, with an overall response rate (ORR) between 6 and $17 \%(21)$.

However, PD-1 blocking agents such as pembrolizumab or nivolumab do not demonstrate antitumor effects in all cancer patients. For instance, pembrolizumab achieved a 19.4\% ORR in treating advanced NSCLC, just over $20 \%$ ORR in treating advanced urothelial cancer, 22\% ORR in treating advanced gastric cancer, and 18.5\% ORR in treating advanced triple negative breast cancer $(13,14,22-24)$. Additionally, adverse effects have been widely observed in patients treated with CTLA-4 or PD-1 blocking agents (25-27). In a PD-1 advanced melanoma clinical trial, drug-related adverse effects were reported in $79 \%$ of patients (107/135), and $13 \%$ of patients (17/135) demonstrated grade 3 or 4 drug-related adverse effects (28). A recent systematic meta-analysis revealed increased rates of hypothyroidism (odds ratio $=7.56,95 \%$ confidence interval 4.53-12.61), pneumonitis (odds ratio $=$ 5.37, 95\% confidence interval 2.73-10.56), colitis (odds ratio $=$ $2.88,95 \%$ confidence interval 1.3-6.37), and hypophysitis (odds ratio $=3.38,95 \%$ confidence interval $1.02-11.08$ ) in cancer patients receiving anti-PD-1 treatments compared with standard treatments (29).

The discovery of additional immunoinhibitory receptors on $\mathrm{T}$ cells creates more options for mono-blockades or combinatorial blockades. T-cell immunoglobulin and mucindomain containing-3 (Tim-3) was first discovered in 2002 
as an IR on $\mathrm{T}$ cells (30). Galectin-9, phosphatidylserine (PtdSer), high mobility group protein B1 (HMGB1), and carcinoembryonic antigen cell adhesion molecule 1 (CEASAM1) are current known ligands that can interact with Tim-3, inhibiting effector T-cell function (31-36). "Killer cell lectin like receptor G1" (KLRG-1) was known as an IR and a T-cell differentiation marker (37-39). As an IR, KLRG-1 can bind with e-cadherin, inhibiting $\mathrm{T}$-cell function via Akt signaling $(40,41)$. KLRG-1 blockade is able to restore the phosphorylation of Akt signaling resulting in the enhancement of proliferative activity of dysfunctional CD8 T cells $(42,43)$. Both B- and T-lymphocyte attenuator (BTLA) and CD160 are able to impair antitumor T-cell responses by binding with herpes virus entry mediator (HVEM) (44-46). CD244 (2B4) can bind with CD48 and has dual roles as a stimulatory receptor and an IR $(47,48)$. The inhibitory function induced through Tcell immunoglobulin and ITIM (TIGIT) was quite similar to that of CTLA-4. The binding between CD226 and CD155 could deliver stimulatory signaling in T cells and NK cells (49). However, TIGIT competitively binds with CD155 with much higher affinity than with CD226, delivering inhibitory signaling to $\mathrm{T}$ cells and NK cells (50). Given the breadth of potential immunoinhibitory pathways available in the tumor microenvironment in addition to PD-1 and CTLA-4, the next critical questions to tackle include the following: (1) Which immunoinhibitory pathway blockade regimens should be combined to enhance and sustain antitumor responses and thus improve cancer patient outcomes? (2) Which inhibitory pathway blockade might elicit severe autoimmunity and thus should be carefully managed?

In this study, we evaluated the potential risks, benefits, and suitability of potential blockade immunotherapy for eight of the most prevalent solid tumors. Considering the potential alteration that radiotherapy or chemotherapy may bring to the expression of IRs on tumor-infiltrating $\mathrm{T}$ cells (TILs), all the patients recruited in our cohort were treatment-naïve, primary cancer individuals ranging from the eight most prevalent cancer types (51). This valuable cohort allows us to comprehensively evaluate the overall expression of key IRs on $T$ cells in patients with multiple types of cancer, aiming to provide useful information for both clinicians who practice blockade immunotherapy on advanced-cancer patients and scientists who devote themselves to characterizing the mechanisms of a specific inhibitory pathway on $\mathrm{T}$ cells. We investigated the surface expression of eight IRs on $\mathrm{T}$ cells in peripheral blood and tumor-infiltrating lymphocytes (TILs). We propose that systemic infusion of blocking agents that target IRs expressed highly in peripheral blood, such as BTLA, might trigger autoimmunity in patients. We observed co-expression of PD-1, Tim-3, and TIGIT on cytotoxic (CD8) TILs in multiple types of cancer, which indicates the phenotype of CD8 TILs for future in vitro analysis of dysfunctional $\mathrm{T}$ cells. Furthermore, we found that a high frequency of Tim-3+ CD8 TILs tended to associate with poorly differentiated cervical cancer. These data suggest that cancer differentiation type, a well-established routine clinical test, represents a potential biomarker for the suitability of Tim-3 blockade immunotherapy.

\section{MATERIALS AND METHODS}

\section{Study Subjects and Ethical Statement}

Fresh surgical samples with paired peripheral blood of primary cancer patients were collected in Beijing You'an Hospital, Capital Medical University, and Xinjiang Tumor Hospital, Xinjiang Medical University. Written informed consent was obtained from all cancer patients. All the patients were diagnosed and confirmed as primary cancer individuals who have not received any anticancer treatments beforehand. Fresh tumor samples were collected from either surgeries or biopsies. All methods were performed in accordance with the relevant guidelines and regulations, with ethical approval obtained from the Oxford Radcliffe Biobank (ORB) research tissue bank ethics committee (OCHRE reference 17/A006; REC reference 09/H0606/5+5), Oxford Tropical Research Ethics Committee (OxTREC reference 587-16), and the First Affiliated Hospital of Xinjiang Medical University Ethics Committee and Beijing You'an Hospital Ethics Committee.

\section{Isolation of Lymphocytes From Blood and Tumor Tissues}

Peripheral blood mononuclear cells (PBMCs) were isolated from fresh heparinized blood by Ficoll-Hypaque density gradient centrifugation. Surgical tumor tissues were immediately transferred to tumor dissociation solution-containing (Miltenyi Biotec, catalog no. 130-095-929) C tube (Miltenyi Biotec, catalog no. 130-093-237). The tissues were then dissected into 1- to 3$\mathrm{mm}$ pieces by sterile surgical scissor (Ethicon, USA). C tubes were placed on Octo-gentle dissociator (Miltenyi Biotec, catalog no. 130-095-937). Human tumor program-1 was performed for the dissociation followed by 20-min incubation on the gentle-mix rotator (Miltenyi Biotec, catalog no. 130-090-753) at $37^{\circ} \mathrm{C}, 5 \%$ $\mathrm{CO}_{2}$ incubator. A 70-nm cell strainer (Sigma-Aldrich, Dorset, $\mathrm{UK}$ ) was then used to purify the intra-tumor or intra-tissue lymphocytes. Further, cells were washed twice in R10 and counted by trypan blue staining.

\section{Multichromatic Flow Cytometry Staining}

From 2012 to 2014, eight-color panels were designed for an ex vivo phenotypic analysis. From 2014 onwards, with an upgraded flow cytometer, a 14-color panel was designed for the surface analysis of multiple IRs on T cells, which allowed us to investigate the co-expression of multiple IRs on TILs. Reviewing the results from the study in the first 2 years, when we upgraded the panel from 2014 onwards, we decided to exclude BTLA and CD160 from the updated panel owing to their low expressions on TILs and to add TIGIT and three T-cell differentiation markers (CD27, CCR7, and CD45RA) to the 14-color panel. Subsequently, we conducted a co-expression analysis in patients with multiple types of cancer. The details of panels and antibodies are listed in Supplementary Table 2. Cells derived from paired tumor and PBMC sample were each initially stained with LIVE/DEAD ${ }^{\circledR}$ Fixable Aqua Dead Cell Stain Kit (Thermo Fisher Scientific) for $20 \mathrm{~min}$ before surface staining with conjugated antibodies in fluorescenceactivated cell sorting (FACS) washing buffer [phosphate-buffered 
saline [PBS] with $0.5 \mathrm{M}$ of ethylenediaminetetraacetic acid [EDTA] and 7.5\% bovine serum albumin [BSA] solution] for another $20 \mathrm{~min}$ and fixed with $1 \times$ CellFix solution (BD Biosciences). Commercial conjugated antibodies used include CD3-Alexa Fluor 700 (344822, BioLegend), CD4FITC (345768, BD Biosciences), CD8-APC-Cy7 (560179, BD Biosciences), CD160-PE-cy7 (341212, BioLegend), BTLA-APC (344510, BioLegend), PD-1-BV650 (564104, BD Biosciences), Tim3-BV421 (345008, BioLegend), TIGIT-PE (12-9500-42, eBioscience), 2B4-APC (329512, BioLegend), KLRG-1-BV605 (138419, BioLegend), CTLA-4-PE-Cy7 (349914, BioLegend), CD27-PERCP5.5 (356407, BioLegend), CCR7-BV711 (353227, BioLegend), and CD45RA-BV785 (304139, BioLegend). The antibody cocktails were tested in advance with or without the use of tumor dissociation solution to ensure proper function. Fluorescence minus one (FMO) controls were applied accordingly in order to properly position gates. In order to ensure the quality of FACS data, we ruled out any tumor samples in which the viable CD3+ TILs were lower than 10,000 cells. The full gating strategy of two 8-color FACS panels and 14-color FACS panel is shown in Supplementary Figures 4-8. The exemplary gates of IRs on T cells are shown in Figures 2A,B, 4A,B.

\section{Statistical Analysis}

Mono-expression analysis and correlation analysis graphs were generated and analyzed in GraphPad v.7 software (Prism). A co-expression analysis was conducted in Simplified Presentation of Incredibly Complex Evaluations (SPICE) software [National Institute of Allergy and Infectious Diseases (NIAID)]. Unless stated otherwise, data are summarized as median \pm s.e.m. Statistically significant differences between two groups were assessed using a two-tailed paired $t$-test, with Wilcoxon adjustments for non-parametrically distributed variables. Differences were considered statistically significant at $P<0.05$, $\left({ }^{*} P<0.0332,{ }^{* *} P<0.0021,{ }^{* * *} P<0.0002\right.$, and $\left.{ }^{* * * *} P<0.0001\right)$.

\section{RESULTS}

\section{Differential Surface Expression of Inhibitory Receptors on T Cells in Blood vs. Tumors From Cancer Patients}

To comprehensively investigate the immunoinhibitory pathways in T cells, we used FACS to analyze the surface expression of eight IRs (PD-1, Tim-3, BTLA, KLRG-1, TIGIT, 2B4, CD160, and CTLA-4) on T cells from peripheral blood and tumors isolated from 131 cancer patients (Supplementary Table 1, Figure 1). Having updated the panel in 2014, removing CD160 and BTLA, and adding TIGIT, the number of patients used for the expression analysis of BTLA and CD160 and that of TIGIT is different from that of other IRs. Details of the overlapping of patients for different IR analyses can be found in Supplementary Table 4. We observed distinct surface expression of IRs on CD8 (cytotoxic) $\mathrm{T}$ cells compared with CD4 T cells from peripheral blood. For instance, $2 \mathrm{~B} 4+$ and KLRG-1+ cells displayed a high frequency in the CD8 circulating T-cell population but an almost five times lower frequency in the population of CD4 circulating $\mathrm{T}$ cells.
In contrast, $\mathrm{PD}-1+$ cells showed a similar and low frequency in circulating CD4 T cells and CD8 T cells. We also observed unique expression patterns in TILs. For instance, although KLRG-1+ cells displayed a relatively low frequency in both CD8 and CD4 TILs, 2B4+ cells accounted for almost $80 \%$ of CD8 TILs.

We also compared the mean frequencies of cells expressing IRs with the mean frequency of PD-1+ cells. Peripheral CD8 T cells showed two-fold higher frequencies of BTLA+, KLRG-1+, TIGIT+, and 2B4+ cells than did PD-1+. Further, CD8 TILs, relative to $\mathrm{PD}-1+$, showed two-fold higher mean frequencies of TIGIT + and 2B4+ cells. Circulating CD4 T cells, relative to PD$1+$, showed higher frequencies of BTLA + and TIGIT + , but only TIGIT+ CD4 TILs were more frequent than PD-1+ CD4 TILs.

Generally, the IRs were expressed more frequently on CD8 T cells compared with CD4 T cells and on TILs compared with circulating $\mathrm{T}$ cells, the exception being BTLA surface expression, which showed the highest frequency on circulating CD4 T cells from patients.

\section{Increased PD-1+ and Tim-3+ but Decreased BTLA+ T-Cell Frequencies Are Observed in Tumors When Compared With Peripheral Blood From Individual Patients Across Multiple Cancer Types}

To determine if the IRs are differentially expressed on TILs vs. peripheral $\mathrm{T}$ cells from individual patients, we used FACS to analyze blood-tumor-matched samples from the 131 primary cancer patients (clinical characteristics are listed in Supplementary Table 1). We detected significantly higher frequencies of PD-1+ and Tim-3+ cells in TILs compared with their counterparts from peripheral blood across eight types of cancer (Figures 2C-F). In contrast, the frequency of BTLA+ cells significantly decreased in CD4 and CD8 TIL populations when compared with the respective peripheral blood populations across multiple cancer types from 74 of these cancer patients (Figures 2G,H, where esophageal, gastric, and colorectal cancers were analyzed together as "digestive tract cancers"). Further, we discovered that the frequencies of $\mathrm{PD}-1+$ and Tim-3+ cells in peripheral T cells positively correlated with their respective frequencies in TILs (Supplementary Figures 1A-D). In contrast, BTLA surface expression in peripheral $\mathrm{T}$ cells and TILs did not correlate (Supplementary Figures 1E,F). Similarly, we observed an increased frequency of TIGIT+ cells in both CD4 and CD8 TILs when compared with $\mathrm{T}$ cells in peripheral blood from lung, cervical, gastric, and colorectal cancer patients; breast and esophageal cancer patients showed a similar trend, but it was not statistically significant (Supplementary Figures 2E,F). In contrast, the frequencies of 2B4+ (Supplementary Figures 2A,B) and CD160+ (Supplementary Figures 2I,J) T cells were similar in peripheral blood and tumor; the frequency of $2 \mathrm{~B} 4+$ cells was high in both peripheral and tumor-infiltrating CD8 T cells (Supplementary Figure 2A).

KLRG-1+ CD8 T cells displayed a lower frequency in tumors compared with peripheral blood from patients with breast, cervical, esophageal, gastric, and colorectal cancers but not 
Blood

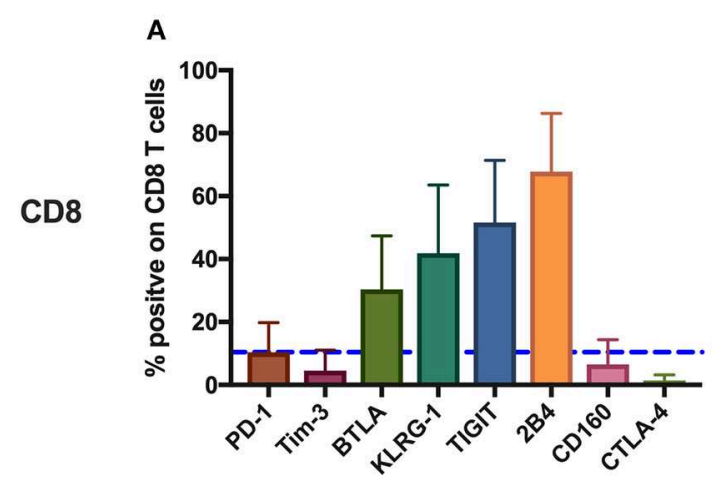

C

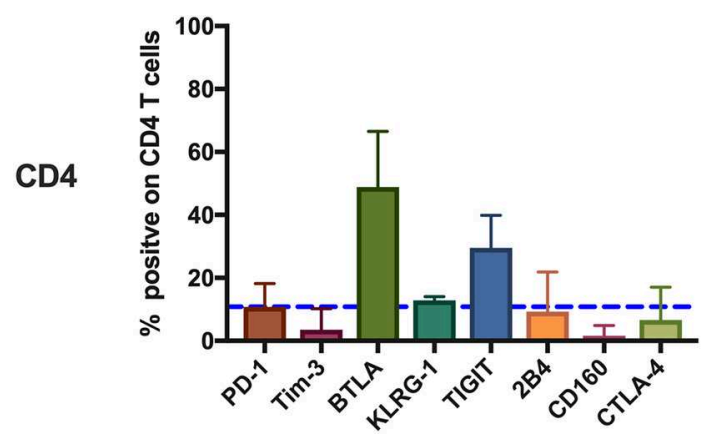

Tumor

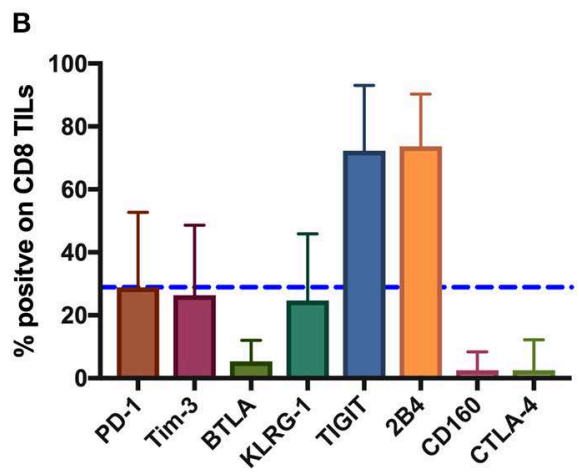

D

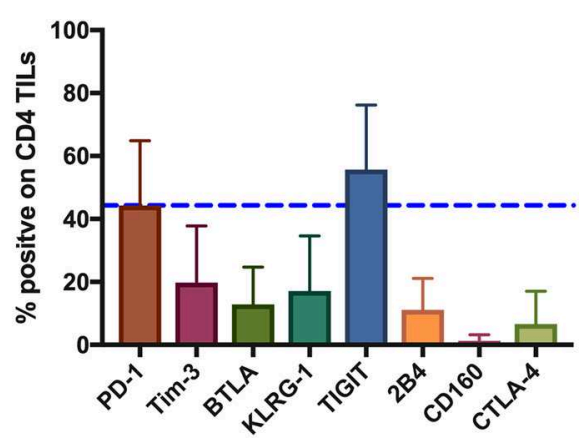

FIGURE 1 | Inhibitory receptors have distinct frequencies in T-cell populations from peripheral blood and tumor tissues from eight types of cancer patients. FACS analysis reveals the frequency of PD-1+, Tim-3+, BTLA+, TIGIT+, 2B4+, CD160+, and CTLA-4+ cells in peripheral CD8 T cells (A), CD8 TILs (B), peripheral CD4 T cells (C), and CD4 TILs (D). T cells were obtained from 131 primary cancer patients, including 38 with breast cancer, 10 with liver cancer, 11 with lung cancer, 9 with esophageal cancer, 14 with gastric cancer, 15 with colorectal cancer, 14 with kidney cancer, and 20 with cervical cancer. The blue dotted line is the mean frequency of PD-1+ cells on each graph. The abovementioned IRs were investigated in three different panels (details are shown in Supplementary Table 2). 131 cancer patients were used for the analysis of PD-1, Tim-3, KLRG-1, and 2B4; 74 cancer patients were used for the analysis of BTLA and CD160; and 67 cancer patients were investigated for the analysis of TIGIT. Details of the overlapping patients and the number of the patients in each cancer cohort are listed in Supplementary Table 4. FACS, fluorescence-activated cell sorting; TILs, tumor-infiltrating lymphocytes; PD-1, programmed cell death protein 1; Tim-3, T-cell immunoglobulin and mucin-domain containing-3; KLRG-1, killer cell lectin like receptor G1; 2B4, CD244; BTLA, B- and T-lymphocyte attenuator.

with kidney, lung, and liver cancers (Supplementary Figure 2C). Although KLRG-1+ CD4 T cells showed a higher frequency in tumors compared with blood from kidney cancer patients, they displayed similar frequencies in blood and tumors from patients with the remaining cancer types (Supplementary Figure 2D).

Notably, we found that cells expressing CTLA-4, the first approved checkpoint blockade target, displayed a generally low frequency $(<20 \%)$ in both CD4 and CD8 T cell populations in the tumor and peripheral blood across eight cancer types. However, multiple cancers showed an increased frequency of CTLA-4+ CD4 T cells, but not CTLA-4+ CD8 T cells, in tumors compared with peripheral blood (Supplementary Figures 2G,H).

\section{Co-expression of Inhibitory Receptors Identifies Shared Subsets of CD8 TILs in Multiple Types of Cancer}

To investigate the co-expression patterns of multiple IRs on CD8 TILs, we focused on PD-1, Tim-3, TIGIT, 2B4, KLRG-1, and CTLA-4. We identified PD-1+TIGIT+2B4+Tim-3+KLRG-1CTLA-4- and PD-1+TIGIT+2B4+Tim-3-KLRG-1-CTLA-4- as dominant shared subsets of CD8 TILs in 64 cancer patients ( 4 with esophageal cancer, 11 with gastric cancer, 12 with colorectal cancer, 7 with breast cancer, 10 with lung cancer, 10 with kidney cancer, and 10 with cervical cancer) (Figures 3A-G). Of note, five liver cancer patients showed a distinct shared subset of 2B4+TIGIT+KLRG-1+1PD-1-Tim-3-CTLA-4- CD8 TILs (Figure 3H). Our co-expression analysis appears to underscore the prominent role of the PD-1, TIGIT, and Tim-3 IRs on CD8 TILs in T-cell exhaustion for multiple types of cancer.

\section{Tim-3 and TIGIT Are Preferentially Expressed on PD-1+ CD8 TILs in Cancer Patients}

The mono-expression and combinatorial expression analyses suggested the importance of PD-1, Tim-3, and TIGIT on CD8 T cells. We specifically investigated the co-expression of Tim3 with PD-1 on CD8 TILs from the same cohort of cancer patients as mentioned in the co-expression analysis of IRs on CD8 TILs. We found that Tim-3+PD-1- CD8 TILs accounted 

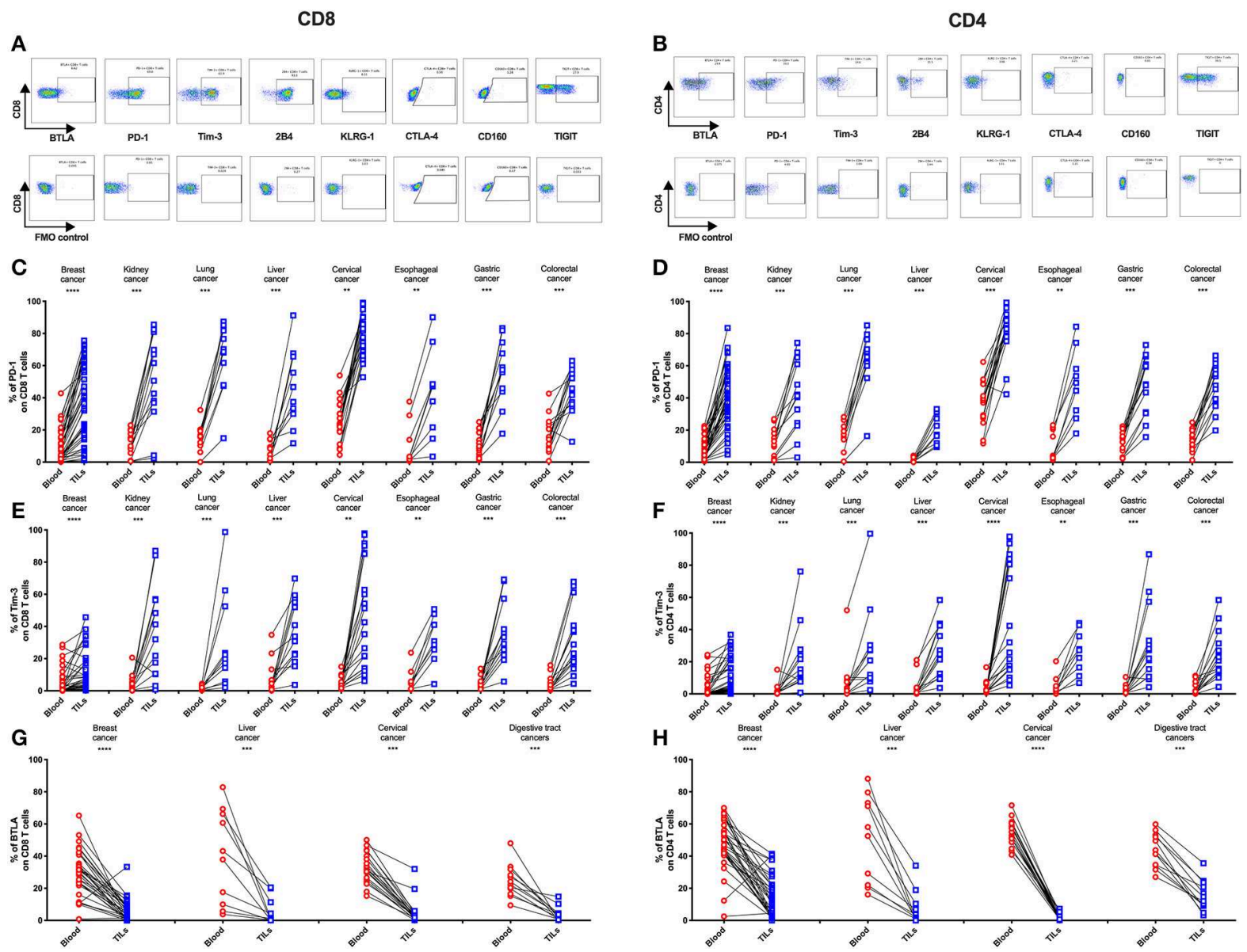

FIGURE 2 | Higher frequencies of PD-1 and Tim-3 but lower frequencies of BTLA in T cells from tumors compared with peripheral blood in matched patient samples. Representative dot plots of eight inhibitory receptors on CD8 (A) and CD4 (B) TILs with FMO controls are shown. The exemplary plots are combined from two 8-color panels of two breast cancer patients. Full gating strategy of two 8-color panels and the 14-color panel is shown in Supplementary Figures 4-8. FACS analysis of PD-1 (C,D) and Tim-3 (E,F) in CD8 (left) and CD4 (right) T cells was performed for 131 blood-tumor-matched primary cancer patients (see Figure $\mathbf{1}$ for details). Analysis of BTLA+ cells in CD8 (G) and CD4 (H) T cells was performed for 74 blood-tumor-matched cancer patients: 32 breast cancer, 10 liver cancer, 20 cervical cancer, and 12 digestive tract cancer (five esophageal cancer, four gastric cancer, and three colorectal cancer). Wilcoxon paired $t$-test was performed to detect the statistical significance $\left({ }^{\star} P<0.0332,{ }^{\star \star} P<0.0021,{ }^{\star \star \star} P<0.0002\right.$, and $\left.{ }^{\star \star \star \star} P<0.0001\right)$. The fraction of cells expressing PD-1, Tim-3, or BTLA on the surface of $\mathrm{T}$ cells in peripheral blood vs. tumor is shown as \%. PD-1, programmed cell death protein 1; Tim-3, T-cell immunoglobulin, and mucin-domain containing-3; BTLA, B-, and T-lymphocyte attenuator; TILs, tumor-infiltrating lymphocytes; FMO, fluorescence minus one; FACS, fluorescence-activated cell sorting.

for $<5 \%$ of bulk CD8 TILs (Figure 4C), whereas PD-1+Tim3- and PD-1+Tim-3+ CD8 TILs accounted for about 10 and around 20\%, respectively, of CD8 TILs (Figure 4C). We then investigated the co-expression of TIGIT and PD-1 on CD8 TILs from the same cohort. Approximately $50 \%$ of CD8 TILs were PD$1+$ TIGIT+, around $20 \%$ were TIGIT+PD-1-, and about $10 \%$ were PD-1+TIGIT- (Figure 4D). Thus, Tim-3 and TIGIT are preferentially expressed on PD-1+ CD8 TILs.

\section{High Tim-3+ CD8 TILs Frequency Associates With Cervical Cancer Differentiation Status but Not Stage}

To determine if the expression of IRs on CD8 TILs associates with any clinical characteristics, we investigated 54 cervical cancer patients of whom 20 are blood-tumor tissue-matched patients and 34 are tumor tissue-only patients in our clinical cohort. In the expression analysis of Tim-3 on CD8 TILs from 20 bloodtumor tissue-matched cervical cancer patients, we detected two sub-clusters, a Tim-3 high-frequency group and a Tim-3 lowfrequency group (Figure 5A). We used the mean frequency of Tim-3 as a cutoff value to classify all cervical cancer patients into two subgroups. Based on the pathological diagnosis, in 54 cervical tumor tissues, the Tim-3 high-frequency subgroup had a higher percentage $(52 \%, 11 / 21)$ of poorly differentiated cancer types than had the Tim-3 low-frequency subgroup (27\%, 9/33) in cervical cancer patients, although we did not detect statistical significance of Tim-3+ CD8 TIL frequency between poorly differentiated and moderately differentiated cervical cancer patients (Figure 5B, Supplementary Figure 3A). When stratifying all the cervical cancer patients by their cancer stage, we did not observe a differential frequency of Tim-3+ CD8 TILs (Supplementary Figure 3B). 


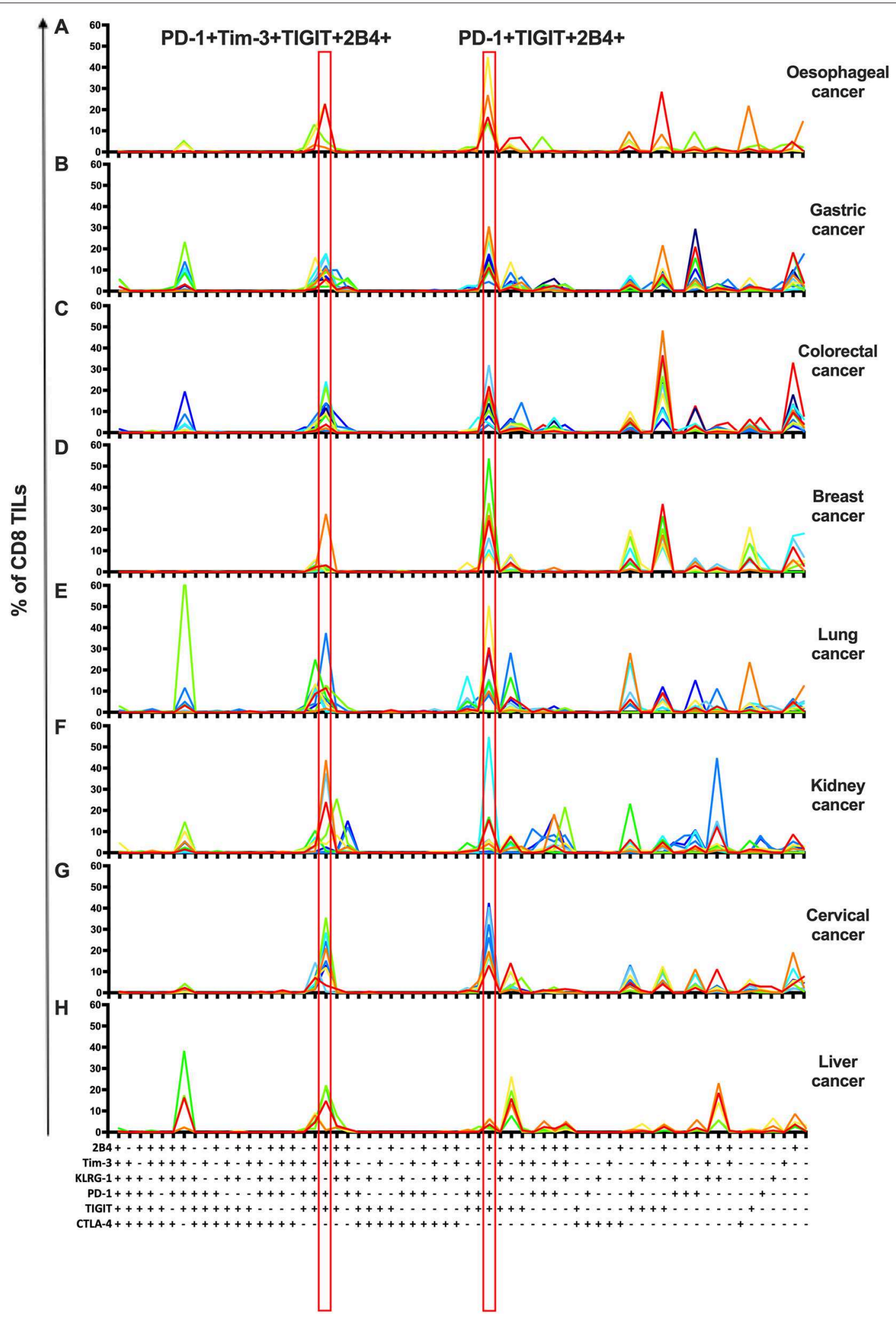

FIGURE 3 | Co-expression analysis identifies shared subsets of CD8 TILs across multiple types of cancer. Simplified Presentation of Incredibly Complex Evaluations (SPICE) analysis was applied to investigate the co-expression of PD-1, Tim-3, 2B4, TIGIT, KLRG-1, and CTLA-4 on CD8 TILs from 69 cancer patients (4 with esophageal cancer, 11 with gastric cancer, 12 with colorectal cancer, 7 with breast cancer, 10 with lung cancer, 10 with kidney cancer, 10 with cervical cancer, and 5 
FIGURE 3 | with liver cancer). With six IRs investigated, there are 64 possible combinations/subsets ( $X$ axis) on CD8 TILs. Single color lines in each graph represent the frequency of different subsets of CD8 TILs from each patient, from eight types of cancer (A-H). Red rectangles highlight the dominant subsets in CD8 TILs shared from 7/8 types of cancer. Co-expression analysis on CD8 TILs was conducted by 14-color panel, utilizing the full gating strategy shown in

Supplementary Figures 8A,B. TILs, tumor-infiltrating lymphocytes; PD-1, programmed cell death protein 1; Tim-3, T-cell immunoglobulin, and mucin-domain containing-3; 2B4, CD244; TIGIT, T-cell immunoglobulin and ITIM; KLRG-1, killer cell lectin like receptor G1; CTLA-4, cytotoxic T-lymphocyte antigen-4; IR, inhibitory receptor.

A

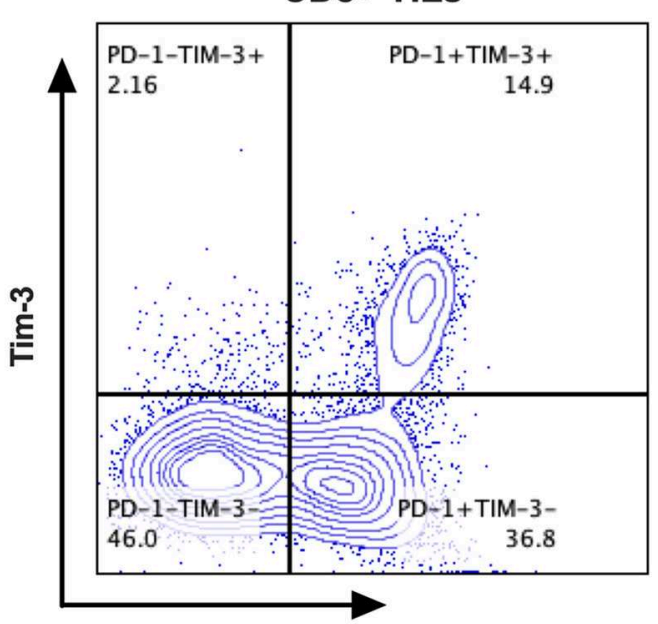

PD-1

C

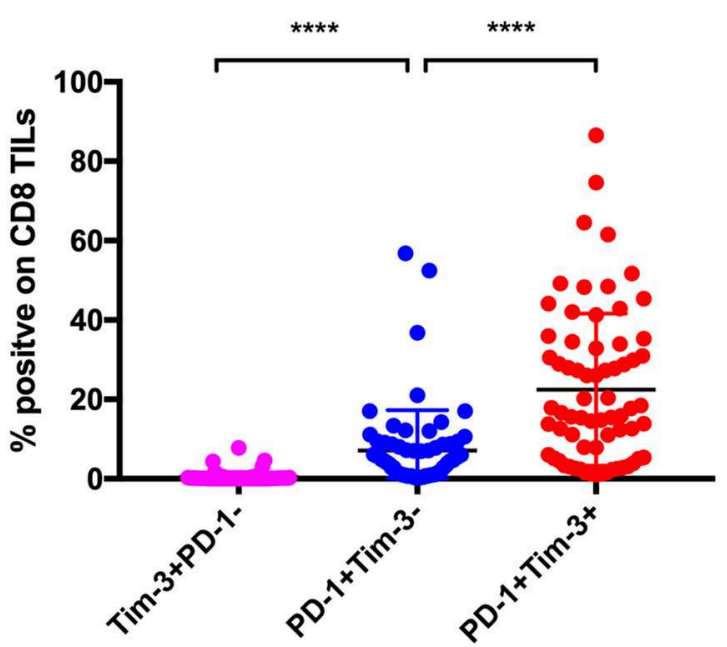

B

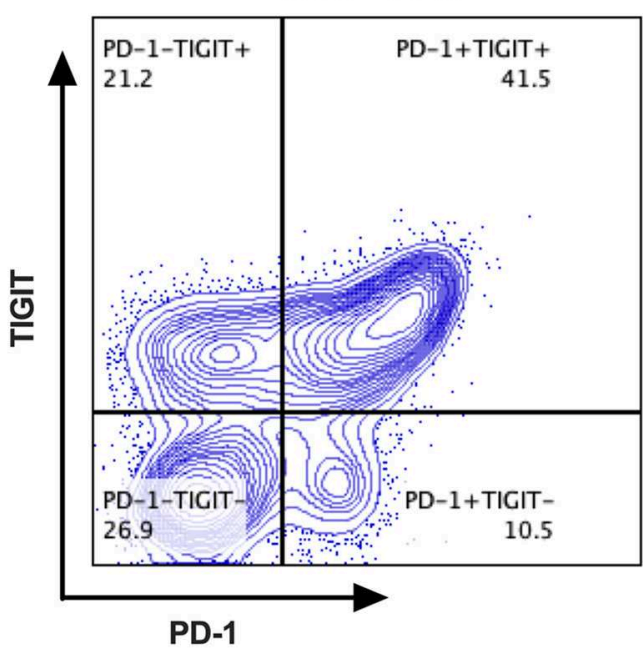

D

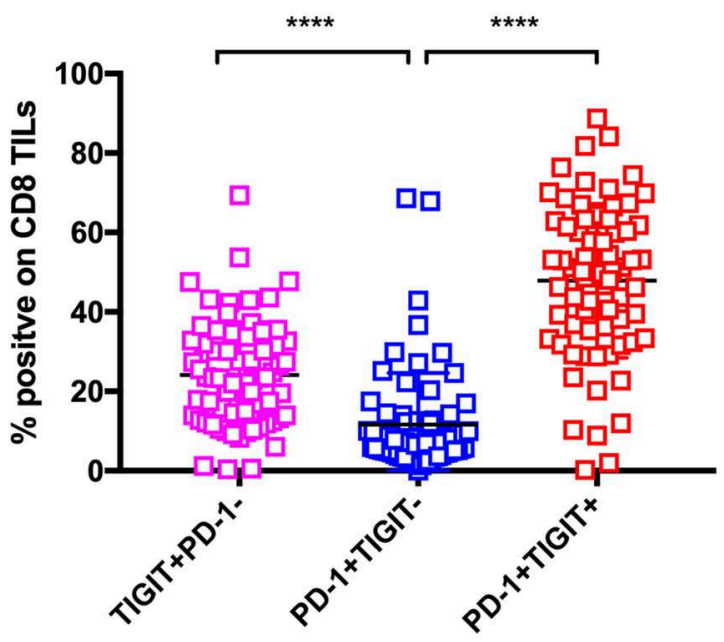

FIGURE 4 | Tim-3 and TIGIT are preferentially expressed on PD-1+ CD8 TILs across multiple types of cancer. Representative dot plots of the co-expression of PD-1 and Tim-3 on CD8 TILs (A) and the co-expression of PD-1 and TIGIT on CD8 TILs (B) from a breast cancer patient are shown. Data were collected utilizing the 14-color panel as mentioned above, with full gating strategy shown in Supplementary Figure 8. (C) FACS analysis of the frequencies of Tim-3+PD-1-, PD-1+Tim-3-, and PD-1+Tim-3+ cells among CD8 TILs from 69 cancer patients (see Figure $\mathbf{3}$ for details). (D) FACS analysis of the frequencies of TIGIT+PD-1-, PD-1+TIGIT-, and PD-1+TIGIT+ cells among CD8 TILs from the same 69 cancer patients. Wilcoxon paired $t$-test was performed to detect the statistical significance $\left({ }^{\star} P<0.0332,{ }^{\star \star} P<0.0021,{ }^{\star * \star} P<0.0002\right.$, and $\left.{ }^{\star \star \star *} P<0.0001\right)$. Tim-3, T-cell immunoglobulin and mucin-domain containing-3; TIGIT, T-cell immunoglobulin and ITIM; TILs, tumor-infiltrating lymphocytes; PD-1, programmed cell death protein 1; FACS, fluorescence-activated cell sorting. 


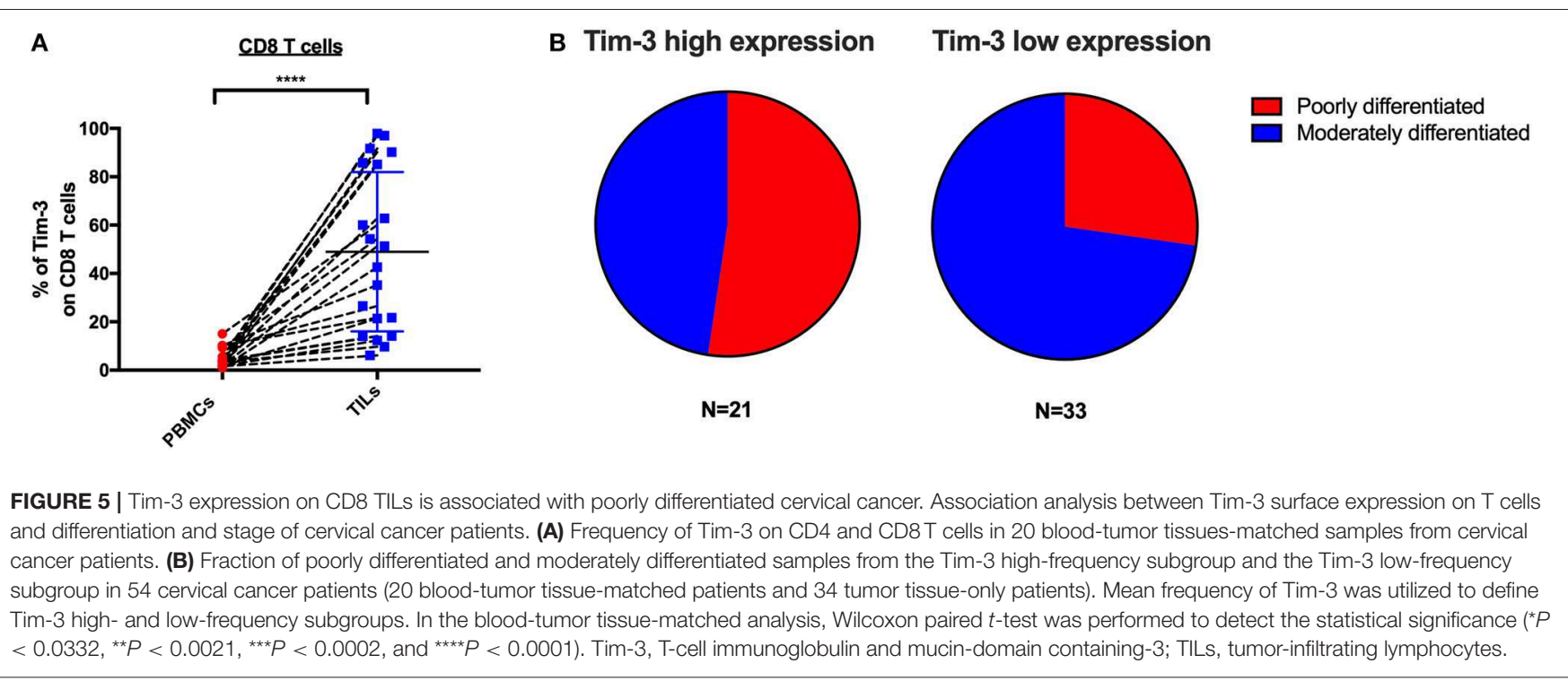

\section{Advanced Differentiation of the Shared PD- 1+Tim-3+TIGIT+2B4+KLRG-1-CTLA-4- CD8 TILs Subset Is Associated With Poorly Differentiated Cervical Cancer}

We further conducted T-cell differentiation analysis of the identified shared subset, PD-1+TIGIT+2B4+Tim-3+KLRG1-CTLA-4- in bulk CD8 TILs from 10 cervical cancer patients. We stratified 10 cervical cancer patients into two subgroups by pathology (five poorly differentiated and five moderately differentiated tumors). CD8 T-cell differentiation stages are assessed by the differential CD27, CCR7, and CD45RA expression (Figure 6C). We found that the majority (over 60\%) of PD-1+TIGIT+2B4+Tim-3+KLRG-1-CTLA-4- CD8 TILs from all $(5 / 5)$ of the poorly differentiated cervical cancer samples displayed an advanced T-cell differentiation phenotype and that only a small fraction (20\%) displayed intermediate T-cell differentiation phenotypes (Figure 6A). Conversely, the majority (60\%) of PD-1+TIGIT+2B4+Tim-3+KLRG-1-CTLA-4CD8 TILs from most (4/5) of the moderately differentiated cervical cancer samples displayed an intermediate T-cell differentiation phenotype, and a minor fraction (40\%) displayed advanced T-cell differentiation phenotypes (Figure 6B). These results suggest that the advanced $\mathrm{T}$-cell differentiation (CD27-CCR7-CD45RA-) of PD-1+TIGIT+2B4+Tim3+KLRG-1-CTLA-4- CD8 TILs is associated with poorly differentiated cervical cancer.

\section{DISCUSSION AND CONCLUSIONS}

We have comprehensively investigated the surface expression of eight IRs on T cells from a valuable clinical cohort comprising only recruited primary cancer, treatment-naïve patients from China and the UK. We demonstrated distinct surface expression of IRs on T cells between peripheral blood and tumor tissues across multiple cancer types, and across CD4 vs. CD8 T cells. $\mathrm{PD}-1+$ and Tim-3 $+\mathrm{T}$ cells were increased in tumors compared with peripheral blood across eight cancer types. These results are in line with previous studies of breast cancer, esophageal cancer, lung cancer, and liver cancer (52-55). Our study also found that BTLA+ T cells were decreased in tumors vs. blood, which is consistent with a recent breast cancer study (52). However, it has been reported in a previous melanoma study that the tumor microenvironment upregulates the expression of BTLA on CD8 TILs and that the co-expression of BTLA and PD-1 marks tumor-specific TILs in melanoma patients (56). In addition, we did not observe any differential expression of any individual IR on TILs in patients among different clinical stages in each type of cancer (data not shown). This is possibly due to the limited number of patients in each stage of an individual cancer type.

Releasing the brakes of IRs can be a double-edged sword: restoring T-cell antitumor responses but inducing immunopathology and breaking self-tolerance $(57,58)$. Patients in clinical trials of nivolumab and pembrolizumab to treat multiple types of cancer showed significantly higher risk of pneumonitis, colitis, hypothyroidism, hypophysitis, and hepatitis $(15,27,59)$. These findings raise concerns about autoimmunity and immunopathology during the development of new blockade immunotherapies. On the basis of our results, we would categorize IRs into four groups, which are summarized in Table 1: group 1 IRs show a lower frequency on T cells in the circulation vs. tumors, such as PD-1 and Tim-3. The immunerelated adverse effects generated by blocking these inhibition pathways are predicted to be manageable. Group 2 IRs show a higher frequency on $\mathrm{T}$ cells in the circulation vs. tumors, such as BTLA and KLRG-1. BTLA is linked with T-cell differentiation and is predominately expressed on naïve $\mathrm{T}$ cells (60). A recent functional study of KLRG-1 in animal models also revealed that after receiving stimulatory and inflammatory signals, KLRG-1+ CD8 T cells downregulated KLRG-1 and differentiated into different memory T-cell linages, with distinctive antivirus and antitumor capacities (61). Group 2 IRs may have less value in checkpoint blockade owing to the likelihood of immune-related 


\section{On PD-1+Tim-3+TIGIT+2B4+CTLA-4-KLRG-1-CD8+'TILs}
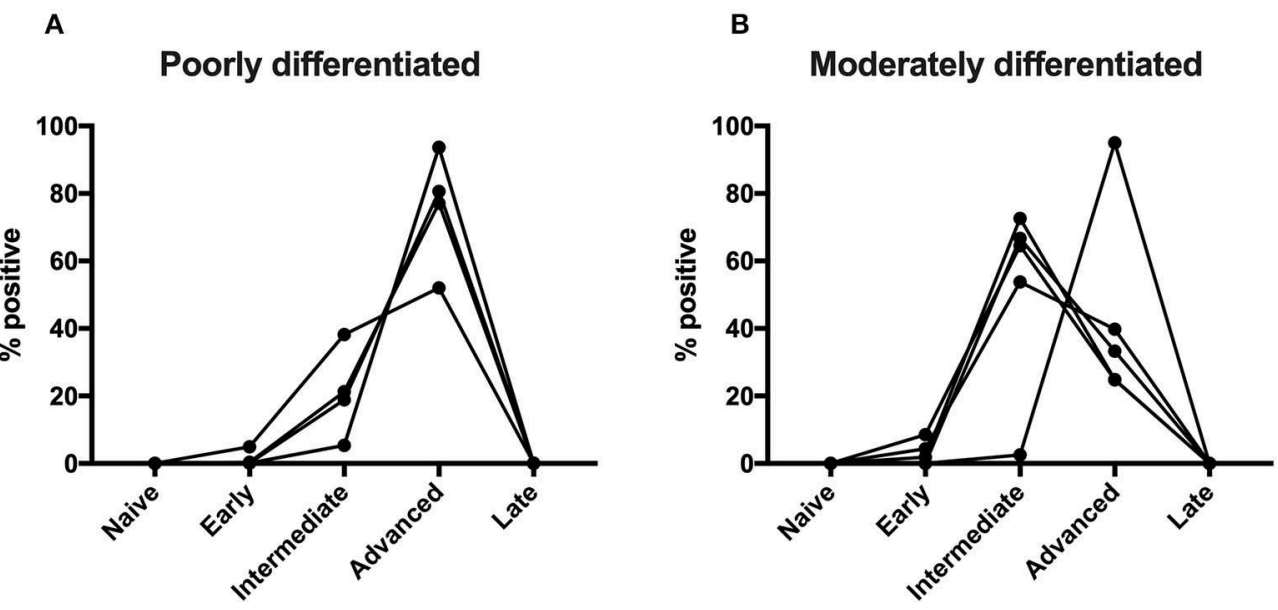

C

\begin{tabular}{|c|c|c|c|c|c|}
\hline & "Naïve" & "Early" & "Intermediate" & "Advanced" & "Late" \\
\hline CD27 & + & + & + & - & - \\
\hline CCR7 & + & + & - & - & - \\
\hline CD45RA & + & - & - & - & + \\
\hline
\end{tabular}

FIGURE 6 | Advanced T cell-differentiated subset in the "shared" subset of CD8 TILs is linked to poorly differentiated cervical cancer. Having gated on PD-1+Tim-3+TIGIT+2B4+CTLA-4-KLRG-1-CD8+ TILs from 10 cervical cancer patients, Boolean combination gating strategy was then used to determine different T-cell differentiation stages by the surface expression of CD27, CCR7, and CD45RA. The frequency of each stage of TILs in the "shared" subset of CD8 TILs was depicted between five poorly differentiated cervical cancer samples (A) and five moderately differentiated cervical cancer samples (B). Five T-cell differentiation stages (C) are "naïve" T cells (CD27+CCR7+CD45RA+), "early" differentiated T cells (CD27+CCR7+CD45RA-), "intermediate" differentiated T cells (CD27+CCR7-CD45RA-), "advanced" differentiated T cells (CD27-CCR7-CD45RA-), and "late" differentiated T cells (CD27-CCR7-CD45RA+). Representative dot plots of CD45RA, CD27, and C-C chemokine receptor type 7 (CCR7) on CD8 TILs with FMO controls are shown in Supplementary Figure 8B. The frequency of five different T-cell differentiation stages was then calculated by the function of Boolean Combination Gates in Flowjo software. TILs, tumor-infiltrating lymphocytes; FMO, fluorescence minus one.

adverse effects resulting from their relatively high expression on peripheral $\mathrm{T}$ cells. However, they may be suitable for genetic manipulation of antigen-specific $\mathrm{T}$ cells, which might limit the immune-related adverse effects caused by non-tumor-specific T cells. Furthermore, local injection of the group 2 IR-blocking agents into the tumor may reduce the immune-related adverse effects when compared to those in systemic infusion. Group 3 IRs show a high frequency on $\mathrm{T}$ cells in both peripheral blood and tumors, such as TIGIT and 2B4. Blocking these inhibition pathways might cause severe immune-related adverse effects, given their expression in peripheral blood $\mathrm{T}$ cells. Similarly to BTLA, these may not be ideal targets for checkpoint blockade that is administered via systemic infusion to patients. However, local injection rather than systemic infusion of blocking agents targeting the TIGIT or 2B4 pathways might minimize the immune-related adverse effects but still benefit patients. Of note, a previous study has shown that 2B4 could act as the ligand rather than receptor on target cells, which interact with CD48 expressing NK cells, activating human NK cells' cytotoxic functions (62). In chronic HCV and HBV infection, it was found that 2B4 was co-expressed with PD-1 on virus-specific CD8 T cells eliciting dysfunctional cytotoxic functions $(6,63)$. TIGIT has been revealed to be upregulated on peripheral $\mathrm{T}$ cells in elderly people, compared with young people, marking exhausted or senescent $\mathrm{T}$ cells in aging-related immunosuppression (64). Therefore, our interpretation is that the high expression level of TIGIT observed in patients from our cohort additively resulted from the presence of cancer and the average ages of patients (56.4 years old, details shown in Supplementary Table 1). Group 4 IRs show a low frequency on $\mathrm{T}$ cells from both peripheral blood and tumors, such as CTLA-4 and CD160. These results are consistent with previous reports that CTLA-4 and CD160 have soluble forms circulating in the blood $(65,66)$. As such, surface staining alone may not identify all CTLA-4+ or CD160+ T cells. In a lung cancer study, a higher frequency of CTLA-4 was found by intracellular staining in CD4 $\mathrm{T}$ cells when compared with those of surface staining in CD4 T cells from the same patients (67). Therefore, it is advisable to intracellularly stain CTLA-4 in T cells. It is also true that we are not sure about the expression of CTLA- 4 on T cells in lymphatic organs such as the spleen or lymph nodes in various locations where $\mathrm{T}$ cells are primed. Furthermore, our study 
TABLE 1 | Summary of four categories of key inhibitory receptors, its expression features, autoimmunity concerns, and clinical implication.

\begin{tabular}{|c|c|c|c|c|}
\hline Group of IRs & Group 1 & Group 2 & Group 3 & Group 4 \\
\hline $\mathbb{I R}$ & PD-1, Tim-3 & BTLA, KLRG-1 & 2B4, TIGIT & CTLA-4, CD160 \\
\hline $\begin{array}{l}\text { Expression in } \\
\text { peripheral blood in } \\
\text { cancer patients }\end{array}$ & + & +++ & +++ & $\begin{array}{l}\text { Express at a negligible level on } \mathrm{T} \\
\text { cells owing to soluble forms }\end{array}$ \\
\hline $\begin{array}{l}\text { Expression in tumor } \\
\text { tissues in cancer } \\
\text { patients }\end{array}$ & +++ & + & +++ & $\begin{array}{l}\text { Express at a negligible level on } \mathrm{T} \\
\text { cells owing to soluble forms }\end{array}$ \\
\hline $\begin{array}{l}\text { Concerns of } \\
\text { autoimmunity }\end{array}$ & Manageable & $\begin{array}{l}\text { Probably capable of inducing } \\
\text { strong immune-related adverse } \\
\text { effects }\end{array}$ & $\begin{array}{l}\text { Probably capable of inducing } \\
\text { strong immune-related adverse } \\
\text { effects }\end{array}$ & $\begin{array}{l}\text { CTLA-4 induces strong } \\
\text { immune-related adverse effects. } \\
\text { The potential immune-related } \\
\text { adverse effects of CD160 } \\
\text { blockade are unknown }\end{array}$ \\
\hline $\begin{array}{l}\text { Implication for } \\
\text { blockade drug } \\
\text { administration }\end{array}$ & $\begin{array}{l}\text { Systemic IV infusion or local } \\
\text { injection }\end{array}$ & Local injection & Local injection & $\begin{array}{l}\text { CTLA- } 4 \text { has been administered } \\
\text { via systemic infusion to patients }\end{array}$ \\
\hline Other applications & $\begin{array}{l}\text { Genetic manipulation of tumor } \\
\text { associated antigen (TAA) specific } \\
\mathrm{T} \text { cells }\end{array}$ & $\begin{array}{l}\text { Genetic manipulation of } \\
\text { TAA-specific T cells }\end{array}$ & $\begin{array}{l}\text { Genetic manipulation of } \\
\text { TAA-specific T cells }\end{array}$ & $\begin{array}{l}\text { Neutralizing antibodies targeting } \\
\text { soluble forms }\end{array}$ \\
\hline
\end{tabular}

only measured the frequency of CTLA-4 on bulk CD4 TILs, but not in different CD4 subsets in the tumor. It was reported that Tregs in the tumor have higher expression of CTLA- 4 than have conventional CD4 TILs (8). More importantly, numerous CTLA-4 blockade animal experiments and human clinical trials have suggested that blocking CTLA- 4 can lead to high-grade immune-related adverse effects, which are of a more severe magnitude, compared with those of PD-1/PD-L1 blockade (68). Therefore, even though high expression of CTLA-4 was not observed on TILs, the vigor of T-cell responses resulting from CTLA-4 blockade may still elicit potent antitumor responses against cancers.

The co-expression of IRs on CD8 TILs in individual cancer patients may inform the design of effective combinatorial blockade regimens. Our co-expression analysis of IRs delineates the shared subset of bulk CD8 TILs as PD-1+TIGIT+2B4+Tim3+KLRG-1-CTLA-4-, supporting the rationale of combinatorial blockade against PD-1, Tim-3, and TIGIT inhibitory pathways. Of note, our interpretation is that in the combinatorial blockade of PD-1, Tim-3, and TIGIT, it is advisable to administer the blocking agents of TIGIT by local injection but not systemic infusion in order to limit the potential immune-related adverse effects. TIGIT has been reported as a co-regulator of the expansion and function of tumorspecific $\mathrm{T}$ cells in advanced melanoma patients, suggesting that co-blockade of PD-1 and TIGIT might elicit potent antitumor responses (69). In 2018, a phase II clinical trial of anti-TIGIT combined with or without PD-L1 blockade in advanced NSCLC patients without epithelial growth factor receptor (EGFR) mutation or anaplastic lymphoma kinase (ALK) translocation was approved (NCT03563716). Further, Tim-3 blockade reinvigorated antitumor T-cell responses in lung cancer patients who developed adaptive resistance to $\mathrm{PD}-1$ blockade (70). From 2015 onwards, the safety and toxicity of antiTim-3 agents such as TSR-022, Sym023, and MBG453 were evaluated in several phase I clinical trials in multiple types of cancer (NCT02817633, NCT03489343, and NCT02608268) (71). Recently, the combinatorial blockade of PD- 1 and Tim-3 in a phase II clinical in liver cancer and multiple solid tumors was initiated in 2018 (NCT03680508 and NCT03744468).

Both our data and a lung cancer study found that Tim3 and TIGIT are preferentially expressed on PD-1+ CD8 TILs in multiple types of cancer, suggesting that combined blockade of these pathways could be effective to treat diverse tumors (72). One liver cancer study indicated that co-blockade of PD-1, CTLA-4, and Tim-3 pathways additively restores the functionality of exhausted CD8 TILs against cancer (54). Another hepatocellular carcinoma (HCC) investigation showed that combinatorial blockade of PD-1 and Tim-3 significantly enhanced the proliferative capacity of CD8 TILs from PD-1 high-expressers (73).

We found that Tim-3+ CD8 TILs might associate with poor cervical cancer differentiation but not stage. Our data further suggest that advanced T-cell differentiation of the shared subset from CD8 TILs in cervical cancer patients also associates with poorly differentiated cervical cancer. In clinical practice, poorly differentiated cancer correlates with poor prognosis. Our findings suggest that cancer differentiation status, which is routinely tested, might be a biomarker to identify cervical cancer patients who would respond to Tim-3 blockade.

To our knowledge, our study represents the first comprehensive analysis of IRs on T cells in blood and tumors from diverse cancer patients. Our findings illuminate the potential risks and benefits of future checkpoint blockades, or of genetic modified T-cell immunotherapy.

\section{DATA AVAILABILITY STATEMENT}

All data generated or analyzed during this study are included in this published article (and its supplementary information files). 


\section{ETHICS STATEMENT}

All procedures involving human subjects, tissues, and clinical data have been performed in accordance with the Declaration of Helsinki. All methods were performed in accordance to the relevant guidelines and regulations, with ethical approval obtained from the Oxford Radcliffe Biobank (ORB) research tissue bank ethics (OCHRE reference 17/A006; REC reference 09/H0606/5+5), Oxford Tropical Research Ethics Committee (OxTREC reference 587-16), and the First Affiliated Hospital of Xinjiang Medical University Ethics Committee and Beijing You'an Hospital Ethics Committee. Written informed consent has been signed by all the participants.

\section{AUTHOR CONTRIBUTIONS}

TD, XL, RW, and NL: study design. XL, XY, PF, MM, CC, YF, LQ, YH, YP, SL, ST, NA, SM, SJ, CV, DM-P, PS, CW, and YZ: data acquisition. XL, PS, NA, SM, NL, CL, and XW: data analysis. XL, TD, VC, CC, and AM: paper writing and edition. All authors read and approved the final manuscript.

\section{FUNDING}

Medical Research Council, UK. John Fell OUP Research Fund; CAMS Oxford CTI seed funding; CAMS Innovation fund 2017PT31043; CAMS Innovation Fund for Medical

\section{REFERENCES}

1. Maini MK, Schurich A. The molecular basis of the failed immune response in chronic HBV: therapeutic implications. J Hepatol. (2010) 52:616-9. doi: 10.1016/j.jhep.2009.12.017

2. Fife BT, Pauken KE, Eagar TN, Obu T, Wu J, Tang Q, et al. Interactions between PD-1 and PD-L1 promote tolerance by blocking the TCR-induced stop signal. Nat Immunol. (2009) 10:1185-92. doi: 10.1038/ni.1790

3. Pauken KE, Wherry EJ. Overcoming $\mathrm{T}$ cell exhaustion in infection and cancer. Trends Immunol. (2015) 36:265-76. doi: 10.1016/j.it.2015.02.008

4. Crespo J, Sun H, Welling TH, Tian Z, Zou W. T cell anergy, exhaustion, senescence, and stemness in the tumor microenvironment. Curr Opin Immunol. (2013) 25:214-21. doi: 10.1016/j.coi.2012.12.003

5. Jiang Y, Li Y, Zhu B. T-cell exhaustion in the tumor microenvironment. Cell Death Dis. (2015) 6:e1792. doi: 10.1038/cddis.2015.162

6. Bengsch B, Martin B, Thimme R. Restoration of HBV-specific CD8+ T cell function by PD-1 blockade in inactive carrier patients is linked to $\mathrm{T}$ cell differentiation. J Hepatol. (2014) 61:1212-9. doi: 10.1016/j.jhep.2014.07.005

7. Jones RB, Ndhlovu LC, Barbour JD, Sheth PM, Jha AR, Long BR, et al. Tim-3 expression defines a novel population of dysfunctional $\mathrm{T}$ cells with highly elevated frequencies in progressive HIV-1 infection. J Exp Med. (2008) 205:2763-79. doi: 10.1084/jem.20081398

8. Montler R, Bell RB, Thalhofer C, Leidner R, Feng Z, Fox BA, et al. OX40, PD-1 and CTLA- 4 are selectively expressed on tumor-infiltrating T cells in head and neck cancer. Clin Transl Immunol. (2016) 5:e70. doi: 10.1038/cti.2016.16

9. Singer M, Wang C, Cong L, Marjanovic ND, Kowalczyk MS, Zhang H, et al. A distinct gene module for dysfunction uncoupled from activation in tumorinfiltrating T cells. Cell. (2016) 166:1500-11 e9. doi: 10.1016/j.cell.2016.08.052

10. Schietinger A, Philip M, Krisnawan VE, Chiu EY, Delrow JJ, Basom RS, et al. Tumor-specific $\mathrm{T}$ cell dysfunction is a dynamic antigen-driven differentiation
Sciences (CIFMS) 2018-I2M-2-002; XL's D Phil study was funded by the China Scholar Council-Nuffield Department of Medicine Oxford University Scholarship; CV's research time was supported by the National Institute for Health Research (NIHR) Oxford Biomedical Research Center (BRC) (Molecular Diagnostics Theme/Multimodal Pathology Subtheme); DM-P was funded by the National Institute for Health Research (NIHR) Oxford Biomedical Research Center (BRC) (Molecular Diagnostics Theme/Multimodal Pathology Subtheme).

\section{ACKNOWLEDGMENTS}

We thank the General Surgery Department, Biomedicine Department and Pathology Department in Beijing You'an Hospital, Capital Medical University; Surgery Department in the Third Affiliated Hospital, Xinjiang Tumor Hospital, Xinjiang Medical University; and Oxford Radcliffe Biobank in the Department of Cellular Pathology, Oxford University Hospitals NHS Trust, UK, for the support and facilitation in transferring human samples and providing clinical information and pathological results.

\section{SUPPLEMENTARY MATERIAL}

The Supplementary Material for this article can be found online at: https://www.frontiersin.org/articles/10.3389/fonc. 2019.01066/full\#supplementary-material

program initiated early during tumorigenesis. Immunity. (2016) 45:389-401. doi: 10.1016/j.immuni.2016.07.011

11. Lipson EJ, Drake CG. Ipilimumab: an anti-CTLA-4 antibody for metastatic melanoma. Clin Cancer Res. (2011) 17:6958-62. doi: 10.1158/1078-0432.CCR-11-1595

12. Prieto PA, Yang JC, Sherry RM, Hughes MS, Kammula US, White DE, et al. CTLA-4 blockade with ipilimumab: long-term follow-up of 177 patients with metastatic melanoma. Clin Cancer Res. (2012) 18:2039-47. doi: 10.1158/1078-0432.CCR-11-1823

13. Bellmunt J, de Wit R, Vaughn DJ, Fradet Y, Lee JL, Fong L, et al. Pembrolizumab as second-line therapy for advanced urothelial carcinoma. N Engl J Med. (2017) 376:1015-26. doi: 10.1056/NEJMoal6 13683

14. Garon EB, Rizvi NA, Hui R, Leighl N, Balmanoukian AS, Eder JP, et al. Pembrolizumab for the treatment of non-small-cell lung cancer. N Engl J Med. (2015) 372:2018-28. doi: 10.1056/NEJMoa1501824

15. Sharma P, Retz M, Siefker-Radtke A, Baron A, Necchi A, Bedke J, et al. Nivolumab in metastatic urothelial carcinoma after platinum therapy (CheckMate 275): a multicentre, single-arm, phase 2 trial. Lancet Oncol. (2017) 18:312-22. doi: 10.1016/S1470-2045(17)30065-7

16. Sorscher S. Pembrolizumab in non-small-cell lung cancer. $N$ Engl J Med. (2017) 376:996-7. doi: 10.1056/NEJMc1615559

17. Chuk MK, Chang JT, Theoret MR, Sampene E, He K, Weis SL, et al. FDA approval summary: accelerated approval of pembrolizumab for second-line treatment of metastatic melanoma. Clin Cancer Res. (2017) 23:5666-70. doi: 10.1158/1078-0432.CCR-16-0663

18. Shu CA, Rizvi NA. Into the clinic with nivolumab and pembrolizumab. Oncologist. (2016) 21:527-8. doi: 10.1634/theoncologist.2016-0099

19. Park JJ, Omiya R, Matsumura Y, Sakoda Y, Kuramasu A, Augustine $\mathrm{MM}$, et al. B7-H1/CD80 interaction is required for the induction and 
maintenance of peripheral T-cell tolerance. Blood. (2010) 116:1291-8. doi: 10.1182/blood-2010-01-265975

20. Butte MJ, Keir ME, Phamduy TB, Sharpe AH, Freeman GJ. Programmed death-1 ligand 1 interacts specifically with the B7-1 costimulatory molecule to inhibit $\mathrm{T}$ cell responses. Immunity. (2007) 27:111-22. doi: 10.1016/j.immuni.2007.05.016

21. Brahmer JR, Tykodi SS, Chow LQ, Hwu WJ, Topalian SL, Hwu P, et al. Safety and activity of anti-PD-L1 antibody in patients with advanced cancer. $N$ Engl J Med. (2012) 366:2455-65. doi: 10.1056/NEJMoa1200694

22. Muro K, Chung HC, Shankaran V, Geva R, Catenacci D, Gupta S, et al. Pembrolizumab for patients with PD-L1-positive advanced gastric cancer (KEYNOTE-012): a multicentre, open-label, phase 1b trial. Lancet Oncol. (2016) 17:717-26. doi: 10.1016/S1470-2045(16)00175-3

23. Nanda R, Chow LQ, Dees EC, Berger R, Gupta S, Geva R, et al. Pembrolizumab in patients with advanced triple-negative breast cancer: phase Ib KEYNOTE-012 study. J Clin Oncol. (2016) 34:2460-7. doi: 10.1200/JCO.2015.64.8931

24. Plimack ER, Bellmunt J, Gupta S, Berger R, Chow LQ, Juco J, et al. Safety and activity of pembrolizumab in patients with locally advanced or metastatic urothelial cancer (KEYNOTE-012): a non-randomised, open-label, phase 1b study. Lancet Oncol. (2017) 18:212-20. doi: 10.1016/S1470-2045(17)30007-4

25. Brahmer JR, Drake CG, Wollner I, Powderly JD, Picus J, Sharfman $\mathrm{WH}$, et al. Phase I study of single-agent anti-programmed death1 (MDX-1106) in refractory solid tumors: safety, clinical activity, pharmacodynamics, and immunologic correlates. J Clin Oncol. (2010) 28:3167-75. doi: 10.1200/JCO.2009.26.7609

26. Nishimura H, Okazaki T, Tanaka Y, Nakatani K, Hara M, Matsumori A, et al. Autoimmune dilated cardiomyopathy in PD-1 receptor-deficient mice. Science. (2001) 291:319-22. doi: 10.1126/science.291.5502.319

27. Robert C, Schachter J, Long GV, Arance A, Grob JJ, Mortier L, et al. Pembrolizumab versus ipilimumab in advanced melanoma. $N$ Engl J Med. (2015) 372:2521-32. doi: 10.1056/NEJMoa1503093

28. Hamid O, Robert C, Daud A, Hodi FS, Hwu WJ, Kefford R, et al. Safety and tumor responses with lambrolizumab (anti-PD-1) in melanoma. N Engl J Med. (2013) 369:134-44. doi: 10.1056/NEJMoa1305133

29. Baxi S, Yang A, Gennarelli RL, Khan N, Wang Z, Boyce L, et al. Immunerelated adverse events for anti-PD-1 and anti-PD-L1 drugs: systematic review and meta-analysis. BMJ. (2018) 360:k793. doi: 10.1136/bmj.k793

30. Monney L, Sabatos CA, Gaglia JL, Ryu A, Waldner H, Chernova T, et al. Th1-specific cell surface protein Tim-3 regulates macrophage activation and severity of an autoimmune disease. Nature. (2002) 415:536-41. doi: $10.1038 / 415536 \mathrm{a}$

31. Zhu C, Anderson AC, Schubart A, Xiong H, Imitola J, Khoury SJ, et al. The Tim-3 ligand galectin-9 negatively regulates T helper type 1 immunity. Nat Immunol. (2005) 6:1245-52. doi: 10.1038/ni1271

32. Freeman GJ, Casasnovas JM, Umetsu DT, DeKruyff RH. TIM genes: a family of cell surface phosphatidylserine receptors that regulate innate and adaptive immunity. Immunol Rev. (2010) 235:172-89. doi: 10.1111/j.0105-2896.2010.00903.x

33. DeKruyff RH, Bu X, Ballesteros A, Santiago C, Chim YL, Lee HH, et al. T cell/transmembrane, Ig, and mucin-3 allelic variants differentially recognize phosphatidylserine and mediate phagocytosis of apoptotic cells. J Immunol. (2010) 184:1918-30. doi: 10.4049/jimmunol.0903059

34. Huang YH, Zhu C, Kondo Y, Anderson AC, Gandhi A, Russell A, et al. CEACAM1 regulates TIM-3-mediated tolerance and exhaustion. Nature. (2015) 517:386-90. doi: 10.1038/nature13848

35. Huang YH, Zhu C, Kondo Y, Anderson AC, Gandhi A, Russell A, et al. Corrigendum: CEACAM1 regulates TIM-3-mediated tolerance and exhaustion. Nature. (2016) 536:359. doi: 10.1038/nature17421

36. Tang D, Lotze MT. Tumor immunity times out: TIM-3 and HMGB1. Nat Immunol. (2012) 13:808-10. doi: 10.1038/ni.2396

37. Gothert JR, Eisele L, Klein-Hitpass L, Weber S, Zesewitz ML, Sellmann L, et al. Expanded CD8 $+\mathrm{T}$ cells of murine and human CLL are driven into a senescent KLRG1+ effector memory phenotype. Cancer Immunol Immunother. (2013) 62:1697-709. doi: 10.1007/s00262-013-1473-z

38. Grundemann C, Schwartzkopff S, Koschella M, Schweier O, Peters C, Voehringer D, et al. The NK receptor KLRG1 is dispensable for virus-induced
$\mathrm{NK}$ and CD8+ T-cell differentiation and function in vivo. Eur J Immunol. (2010) 40:1303-14. doi: 10.1002/eji.200939771

39. Hofmann M, Schweier O, Pircher H. Different inhibitory capacities of human and mouse KLRG1 are linked to distinct disulfide-mediated oligomerizations. Eur J Immunol. (2012) 42:2484-90. doi: 10.1002/eji.201142357

40. de Jong R, Stokkers P, Lamme E, Kool JM, Borst F, Brouwer M, et al. Regulation of T-cell differentiation by $\mathrm{CD} 2$ and $\mathrm{CD} 28$ accessory molecules. Immunology. (1991) 74:175-82.

41. Hombach A, Tillmann T, Jensen M, Heuser C, Sircar R, Diehl V, et al. Specific activation of resting $\mathrm{T}$ cells against tumour cells by bispecific antibodies and CD28-mediated costimulation is accompanied by Th1 differentiation and recruitment of MHC-independent cytotoxicity. Clin Exp Immunol. (1997) 108:352-7. doi: 10.1046/j.1365-2249.1997.3481245.x

42. Henson SM, Akbar AN. KLRG1-more than a marker for T cell senescence. Age. (2009) 31:285-91. doi: 10.1007/s11357-009-9100-9

43. Henson SM, Franzese O, Macaulay R, Libri V, Azevedo RI, Kiani-Alikhan S, et al. KLRG1 signaling induces defective Akt (ser473) phosphorylation and proliferative dysfunction of highly differentiated CD8+ T cells. Blood. (2009) 113:6619-28. doi: 10.1182/blood-2009-01-199588

44. Kaye J. CD160 and BTLA: LIGHTs out for CD4+ T cells. Nat Immunol. (2008) 9:122-4. doi: 10.1038/ni0208-122

45. Murphy KM, Nelson CA, Sedy JR. Balancing co-stimulation and inhibition with BTLA and HVEM. Nat Rev Immunol. (2006) 6:671-81. doi: $10.1038 /$ nri1917

46. Freeman GCGJ. The CD160, BTLA, LIGHT/HVEM pathway: a bidirectional switch regulating T-cell activation. Immunol Rev. (2009) 229:244-58. doi: $10.1111 / j .1600-065 X .2009 .00783 . x$

47. Chuang SS, Kumaresan PR, Mathew PA. 2B4 (CD244)-mediated activation of cytotoxicity and IFN-gamma release in human NK cells involves distinct pathways. J Immunol. (2001) 167:6210-6. doi: 10.4049/jimmunol.167.11.6210

48. Aldy KN, Horton NC, Mathew PA, Mathew SO. 2B4+ CD8+ T cells play an inhibitory role against constrained HIV epitopes. Biochem Biophys Res Commun. (2011) 405:503-7. doi: 10.1016/j.bbrc.2011.01.062

49. Bottino C, Castriconi R, Pende D, Rivera P, Nanni M, Carnemolla B. Identification of PVR (CD155) and nectin-2 (CD112) as cell surface ligands for the human DNAM-1 (CD226) activating molecule. J Exp Med. (2003) 198:557-67. doi: 10.1084/jem.20030788

50. Chan CJ, Martinet L, Gilfillan S, Souza-Fonseca-Guimaraes F, Chow MT, Town L, et al. The receptors CD96 and CD226 oppose each other in the regulation of natural killer cell functions. Nat Immunol. (2014) 15:431-8. doi: $10.1038 /$ ni.2850

51. Chacon JA, Schutsky K, Powell DJ. The impact of chemotherapy, radiation and epigenetic modifiers in cancer cell expression of immune inhibitory and stimulatory molecules and anti-tumor efficacy. Vaccines. (2016) 4:43. doi: 10.3390 /vaccines 4040043

52. Egelston CA, Avalos C, Tu TY, Simons DL, Jimenez G, Jung JY, et al. Human breast tumor-infiltrating $\mathrm{CD} 8(+) \mathrm{T}$ cells retain polyfunctionality despite $\mathrm{PD}-1$ expression. Nat Commun. (2018) 9:4297. doi: 10.1038/s41467-018-06653-9

53. Xie J, Wang J, Cheng S, Zheng L, Ji F, Yang L, et al. Expression of immune checkpoints in T cells of esophageal cancer patients. Oncotarget. (2016) 7:63669-78. doi: 10.18632/oncotarget.11611

54. Zhou G, Sprengers D, Boor PPC, Doukas M, Schutz H, Mancham S, et al. Antibodies against immune checkpoint molecules restore functions of tumorinfiltrating T cells in hepatocellular carcinomas. Gastroenterology. (2017) 153:1107-19 e10. doi: 10.1053/j.gastro.2017.06.017

55. Tassi E, Grazia G, Vegetti C, Bersani I, Bertolini G, Molla A, et al. Early effector $\mathrm{T}$ lymphocytes coexpress multiple inhibitory receptors in primary non-small cell lung cancer. Cancer Res. (2017) 77:851-61. doi: 10.1158/0008-5472.CAN-16-1387

56. Fourcade J, Sun Z, Pagliano O, Guillaume P, Luescher IF, Sander C, et al. $\mathrm{CD} 8(+) \mathrm{T}$ cells specific for tumor antigens can be rendered dysfunctional by the tumor microenvironment through upregulation of the inhibitory receptors BTLA and PD-1. Cancer Res. (2012) 72:887-96. doi: 10.1158/0008-5472.CAN-11-2637

57. Corrigan-Curay J, Kiem HP, Baltimore D, O’Reilly M, Brentjens RJ, Cooper L, et al. T-cell immunotherapy: looking forward. Mol Ther. (2014) 22:1564-74. doi: $10.1038 / \mathrm{mt} .2014 .148$ 
58. Mahoney KM, Rennert PD, Freeman GJ. Combination cancer immunotherapy and new immunomodulatory targets. Nat Rev Drug Discov. (2015) 14:561-84. doi: 10.1038/nrd4591

59. Topalian SL, Hodi FS, Brahmer JR, Gettinger SN, Smith DC, McDermott DF, et al. Safety, activity, and immune correlates of anti-PD-1 antibody in cancer. N Engl J Med. (2012) 366:2443-54. doi: 10.1056/NEJMoa1200690

60. Baitsch L, Legat A, Barba L, Fuertes Marraco SA, Rivals JP, Baumgaertner P, et al. Extended co-expression of inhibitory receptors by human CD8 T-cells depending on differentiation, antigen-specificity and anatomical localization. PLoS ONE. (2012) 7:e30852. doi: 10.1371/journal.pone.0030852

61. Herndler-Brandstetter D, Ishigame H, Shinnakasu R, Plajer V, Stecher C, Zhao J, et al. KLRG1(+) effector CD8(+) T cells lose KLRG1, differentiate into all memory $\mathrm{T}$ cell lineages, and convey enhanced protective immunity. Immunity. (2018) 48:716-29 e8. doi: 10.1016/j.immuni.2018.03.015

62. Messmer B, Eissmann P, Stark S, Watzl C. CD48 stimulation by 2B4 (CD244)expressing targets activates human NK cells. J Immunol. (2006) 176:4646-50. doi: 10.4049/jimmunol.176.8.4646

63. Bengsch B, Seigel B, Ruhl M, Timm J, Kuntz M, Blum HE, et al. Coexpression of PD-1, 2B4, CD160 and KLRG1 on exhausted HCV-specific CD8+ T cells is linked to antigen recognition and T cell differentiation. PLoS Pathog. (2010) 6:e1000947. doi: 10.1371/journal.ppat.1000947

64. Song Y, Wang B, Song R, Hao Y, Wang D, Li Y, et al. T-cell Immunoglobulin and ITIM domain contributes to CD8(+) T-cell immunosenescence. Aging Cell. (2018) 17:e12716. doi: 10.1111/acel.12716

65. Fons P, Chabot S, Cartwright JE, Lenfant F, L'Faqihi F, Giustiniani J, et al. Soluble HLA-G1 inhibits angiogenesis through an apoptotic pathway and by direct binding to CD160 receptor expressed by endothelial cells. Blood. (2006) 108:2608-15. doi: 10.1182/blood-2005-12-019919

66. Linsley. CTLA-4 is a second receptor for the B cell acitvation antigen B7. J Exp Med. (1991) 174:561. doi: 10.1084/jem.174.3.561

67. Kwiecien I, Stelmaszczyk-Emmel A, Polubiec-Kownacka M, Dziedzic D, Domagala-Kulawik J. Elevated regulatory $\mathrm{T}$ cells, surface and intracellular CTLA-4 expression and interleukin-17 in the lung cancer microenvironment in humans. Cancer Immunol Immunother. (2017) 66:161-70. doi: 10.1007/s00262-016-1930-6
68. Chen L, Han X. Anti-PD-1/PD-L1 therapy of human cancer: past, present, and future. J Clin Invest. (2015) 125:3384-91. doi: 10.1172/JCI80011

69. Chauvin JM, Pagliano O, Fourcade J, Sun Z, Wang H, Sander C, et al. TIGIT and PD-1 impair tumor antigen-specific CD8(+) $\mathrm{T}$ cells in melanoma patients. J Clin Invest. (2015) 125:2046-58. doi: 10.1172/JCI 80445

70. Koyama S, Akbay EA, Li YY, Herter-Sprie GS, Buczkowski KA, Richards WG, et al. Adaptive resistance to therapeutic PD-1 blockade is associated with upregulation of alternative immune checkpoints. Nat Commun. (2016) 7:10501. doi: 10.1038/ncomms10501

71. Zahavi DJ, Weiner LM. Targeting multiple receptors to increase checkpoint blockade efficacy. Int J Mol Sci. (2019) 20:E158. doi: 10.3390/ijms200 10158

72. Thommen DS, Koelzer VH, Herzig P, Roller A, Trefny M, Dimeloe S, et al. A transcriptionally and functionally distinct PD-1(+) CD8(+) T cell pool with predictive potential in non-small-cell lung cancer treated with PD-1 blockade. Nat Med. (2018) 24:994-1004. doi: 10.1038/s41591-018-0057-z

73. Kim H-D, Song G-W, Park S, Jung MK, Kim MH, Kang HJ, et al. Association between expression level of PD1 by tumor-infiltrating CD8+ T cells and features of hepatocellular carcinoma. Gastroenterology. (2018) 155:193650.e17. doi: 10.1053/j.gastro.2018.08.030

Conflict of Interest: The authors declare that the research was conducted in the absence of any commercial or financial relationships that could be construed as a potential conflict of interest.

Copyright (C) 2019 Li, Wang, Fan, Yao, Qin, Peng, Ma, Asley, Chang, Feng, $H u$, Zhang, Li, Fanning, Jones, Verrill, Maldonado-Perez, Sopp, Waugh, Taylor, Mcgowan, Cerundolo, Conlon, McMichael, Lu, Wang, Li and Dong. This is an open-access article distributed under the terms of the Creative Commons Attribution License (CC BY). The use, distribution or reproduction in other forums is permitted, provided the original author(s) and the copyright owner(s) are credited and that the original publication in this journal is cited, in accordance with accepted academic practice. No use, distribution or reproduction is permitted which does not comply with these terms. 